\title{
Fake Finger Detection Based on Thin-Plate Spline Distortion Model
}

\author{
Yangyang Zhang, Jie Tian, Xinjian Chen, Xin Yang, and Peng Shi \\ Center for Biometrics and Security Research, Key Laboratory of Complex Systems \\ and Intelligence Science, Institute of Automation, Chinese Academy of Sciences, \\ Graduate School of the Chinese Academy of Sciences, \\ P.O. Box 2728 Beijing 100080 China \\ tian@ieee.org, jie.tian@ia.ac.cn \\ http://www. fingerpass.net
}

\begin{abstract}
This paper introduces a novel method based on the elasticity analysis of the finger skin to discriminate fake fingers from real ones. We match the fingerprints before and after special distortion and gained their corresponding minutiae pairs as landmarks. The thin-plate spline (TPS) model is used to globally describe the finger distortion. For an input finger, we compute the bending energy vector by the TPS model and calculate the similarity of the bending energy vector to the bending energy fuzzy feature set. The similarity score is in the range $[0,1]$, indicating how much the current finger is similar to the real finger. The method realizes fake finger detection based on the normal steps of fingerprint processing without special hardware, so it is easily implemented and efficient. The experimental results on a database of real and fake fingers show that the performance of the method is available.
\end{abstract}

Keywords: fake finger, distortion, Thin-plate Spline model, bending energy vector, fuzzy feature set.

\section{Introduction}

Fingerprints have been increasingly utilized to realize individual identification in the civilian's daily life due to the uniqueness, permanence and universality. On the other side, there are still some potential dangerous in fingerprint recognition systems. One of them is caused by fake or artificial fingers, which is crucial for authentication based on fingerprint systems and decreases their security. Many fingerprint capture devices are probably deceived by well-duplicated fake fingers [10]. Therefore it is necessary to detect fake fingers in the fingerprint system, which can ensure that only live fingerprints are capable of generating templates for enrollment and identification. Some approaches have been proposed to resolve the problem [1], [2]. R. Derakhshani et al. [1] utilized detection of a perspiration pattern over the fingertip to identify the fingerprint vitality. Nevertheless, the method experience difficulties in cases of perspiration disorders (too wet or dry) and other abnormal skin conditions. A. Antonelli et al. [2] proposed an approach based on the analysis of finger skin elasticity. They captured a sequence of fingerprints at high frame rate (at least $20 \mathrm{fps}$ ) and tracked the location of each block to 
calculate the DistortionCode. However, it needs special fingerprint capture device and the location tracking among so many frames is time-consuming.

Different from the previous methods, we utilize the Thin-Plate Spline model to globally approach the finger distortion. During the capturing process, the finger was pressed against the sensor surface and deformed according to the force. Under the same pressure, the structure and materials of the fingers determine the distortion of fingerprints. For live fingers, the special way they distort is related to the elasticity of human skin, and the position and shape of the finger bone. It is difficult to simulate the live fingers precisely. Therefore when pressed against the surface of the capture sensor, the fake and real fingers distort in different ways. The distortion is one of the difficult problems in fingerprint recognition, but here it is utilized to discriminate live fingers from fake ones.

We match the fingerprints before and after special distortion to gain their corresponding minutiae pairs as landmarks and use the thin-plate spline (TPS) model to globally describe the finger distortion. The bending energy vector of the TPS model is employed to determine the nature of the finger, and to the best of our knowledge this is the first time for using TPS model in fake finger detection. For an input finger, we firstly compute its bending energy vector of the TPS model, then calculate the similarity of the bending energy vector to the trained bending energy fuzzy feature set. The similarity score is in the range $[0,1]$, indicating how much the current finger is similar to the real one (1 means real finger).

The rest of this paper is organized as follows: section 2 describes the proposed approach, section 3 reports the experiments carried out to validate the new technique, and section 4 draws our conclusion.

\section{Fake Finger Detection Based on TPS Distortion Model}

Previous works [1], [2] show that generally fake fingers are more rigid than skin and the deformation is lower even if made of highly elastic materials. The elasticity is the basis for discriminating fake fingers from real ones. When a real finger moves on the scanner surface, it produces larger distortion than the fake fingers. We use the Thin-plate Spline (TPS) model to describe the finger distortion.

\subsection{Fingerprints Capture and Landmarks Extraction}

In order to describe the distortions produced by the finger, the user is required to firstly place a finger onto the scanner surface without any superficial tension, then to apply some pressure in four directions: $0 \mathrm{deg}$, 90deg, $180 \mathrm{deg}$ and $270 \mathrm{deg}$ respectively. A sequence of acquired fingerprints are captured for each finger including the natural fingerprint $\mathrm{F} 0$ and the distorted ones $\{\mathrm{F} 1, \mathrm{~F} 2, \mathrm{~F} 3, \mathrm{~F} 4\}$.

Before the computation of the TPS distortion model, the acquired fingerprint images are enhanced by the Hong's method [3] and analyzed to extract relevant features related to skin distortion. When the fingerprint is distorted, its minutiae, as the most popular local features in the fingerprint, have different removal according to their locations. If the minutiae distribute almost symmetrically all over the fingerprint, their displacement can represent the global distortion. In Ref. [4], a robust method is proposed to match the distorted fingerprints based on the local triangle features set. During the matching 


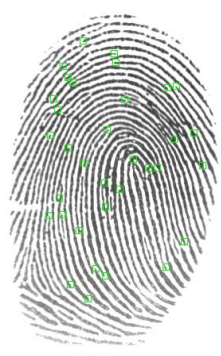

(a)

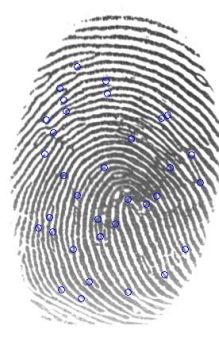

(b)

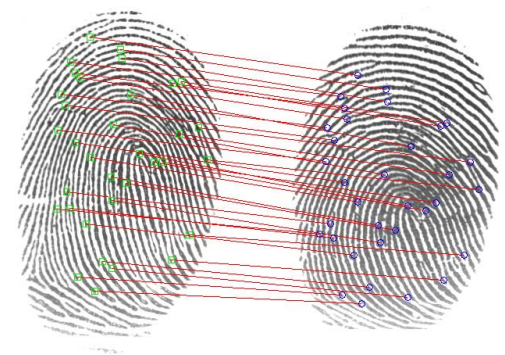

(c)

Fig. 1. (a) fingerprint $\mathrm{F} 0$ with extract minutiae; (b) fingerprint $\mathrm{F} 3$ with extracted minutiae; (c) the two fingerprints with corresponding minutiae paired

process, we can obtain a series of paired minutiae from two fingerprints before and after distortion. In the following, we utilize the paired minutiae as the landmark points to compute the parameters of TPS model. The process of the landmarks extraction is shown in Fig.1

\subsection{Bending Energy Vector Calculation Based on TPS Model}

The thin-plate spline (TPS) model calculate a 2D-2D map based on the arbitrary sets of corresponding points and is able to represent elastic deformations. A.M.Bazen et al. [5] and A. Ross et al. [6] applied thin-plate spline to model the elastic deformation of fingerprints. Since the live fingers have different distorted characteristics from fake ones, their TPS distorted features should have different characters under the same distortion condition caused by the same directional pressure. Given the displacements of a number of landmark points, the TPS model interpolates those points, while maintaining maximal smoothness. At each landmark point $P_{i}$, the displacement is represented by an additional z-coordinate. For each point $(x, y)$ in original image, the TPS model describes the corresponding coordinates $\left(x^{\prime}, y^{\prime}\right)$ in transformed image as $\left(f_{x}(x, y), f_{y}(x, y)\right)$. The bending energy is given by the integral of the second-order partial derivatives over the entire surface and can be minimized by solving a set of linear equations. The TPS model for one of the transformed coordinates is given by the parameter vectors $a$ and $w$ :

$$
f(x, y)=a_{1}+a_{2} x+a_{3} y+\sum_{i=1}^{n} w_{i} \delta\left(\left|P_{i}-(x, y)\right|\right)
$$

where the function $\delta(r)$ is adopted as the root function specially, $\delta(r)=-r^{2} * \log \left(r^{2}\right)$, $a$ defines the affine part of the transformation, $w$ gives an additional non-linear deformation, $P_{i}$ is the $i$ th landmark and $n$ is the number of landmarks.

In fake finger detection, we take the paired minutiae as landmarks. However, the locations of landmarks maybe displaced a few pixels and false local matching maybe introduced because of the mistake in minutiae extraction algorithm. We use the approximating thin-plate splines model [11] to introduce some insensitivity to these 
errors. It allows a smoother transformation instead of entirely interpolating all the landmarks.

$$
Q=(K+\lambda I) * W+P * \alpha
$$

where $I$ is $n * n$ identify matrix, $\lambda$ refers to the weight of the optimization of landmark distance and smoothness. If we adopts large $\lambda$, only the affine transformation is left.

The TPS parameters $W$ and $\alpha$ that minimize the bending energy satisfy the following linear equation:

$$
\begin{aligned}
& {\left[\begin{array}{cc}
q_{x, 1} & q_{y, 1} \\
\vdots & \vdots \\
q_{x, n} & q_{y, n}
\end{array}\right]=\left[\begin{array}{cccc}
\lambda & \delta\left(r_{12}\right)+\lambda & \cdots & \delta\left(r_{1 n}\right)+\lambda \\
\delta\left(r_{21}\right)+\lambda & \cdots & \cdots & \delta\left(r_{2 n}\right)+\lambda \\
\cdots & \cdots & \cdots & \cdots \\
\delta\left(r_{n 1}\right)+\lambda & \delta\left(r_{n 2}\right)+\lambda & \cdots & \lambda
\end{array}\right]\left[\begin{array}{cc}
w_{x, 1} w_{y, 1} \\
\vdots & \vdots \\
w_{x, n} & w_{y, n}
\end{array}\right]} \\
& +\left[\begin{array}{ccc}
1 & p_{x, 1} & p_{y, 1} \\
\vdots & \vdots & \vdots \\
1 & p_{x, n} & p_{y, n}
\end{array}\right]\left[\begin{array}{cc}
a_{x, 1} & a_{y, 1} \\
a_{x, 2} & a_{y, 2} \\
a_{x, 3} & a_{y, 3}
\end{array}\right]
\end{aligned}
$$

where $n$ is the number of landmarks, $r_{i j}=\left|P_{i}-P_{j}\right|$ represents the distance between the landmarks $i$ and $j, P_{i}=\left(p_{x, i}, p_{y, i}\right)$ is the $i t h$ landmark in the natural image, $Q_{i}=$ $\left(q_{x, i}, q_{y, i}\right)$ is the corresponding point in the distorted image.

When applied to fake finger detection, the TPS model must be attached with some modification. The affine part of typical TPS model defined by the matrix $\alpha$ allows the image to translate, rotate and scale. When the fingerprint distorts, its affine transformation can be defined by the vector $\Delta T=(\Delta x, \Delta y, \Delta \theta)$, where $\Delta \theta$ is the rotation angle and $(\Delta x, \Delta y)$ are the translations in $x$ and $y$ coordinates. We realize the global alignment of two fingerprints based on multiple reference minutiae [9]. Then we calculate the transformation matrix $\alpha$ as :

$$
\alpha=\left[\begin{array}{cc}
\Delta x \sin (\Delta \theta) & \cos (\Delta \theta) \\
\Delta y \cos (\Delta \theta) & -\sin (\Delta \theta)
\end{array}\right]
$$

The global bending energy $E$ is calculated as the sum of the bending energy of $\mathrm{X}$ and $Y$ transformed coordinates, which are respectively denoted by $E_{x}$ and $E_{y}$. According to [8], the bending energy for one of the coordinates is estimated as following:

$$
E_{x}=\iint_{R^{2}}\left(\left(\frac{\partial^{2} f_{x}}{\partial x^{2}}\right)^{2}+2 *\left(\frac{\partial^{2} f_{x}}{\partial x \partial y}\right)^{2}+\left(\frac{\partial^{2} f_{x}}{\partial y^{2}}\right)^{2}\right) d x d y
$$

The value of bending energy $E_{x}$ is proportional to $W_{x}^{T} K W_{x}$, where $W_{x}=(K+$ $\lambda I)^{-1}\left(Q_{x}-P_{x} * \alpha_{x}\right)$. The bending energy is zero only when all the components of $W$ are zero. In this case, there is just rigid transformation between two fingerprints.

We compute the bending energy of the finger in four directions: $0 \mathrm{deg}, 90 \mathrm{deg}, 180$ deg and $270 \mathrm{deg}$ respectively. The bending energy vector $V$ is composed of these four bending energy defined as $E_{0}, E_{90}, E_{180}, E_{270}$. The obtained bending energy vector characterizes the deformation of a finger under a specific condition. Fig. 2 illustrates the main steps of the bending energy vector computation. A sequence of fingerprints 


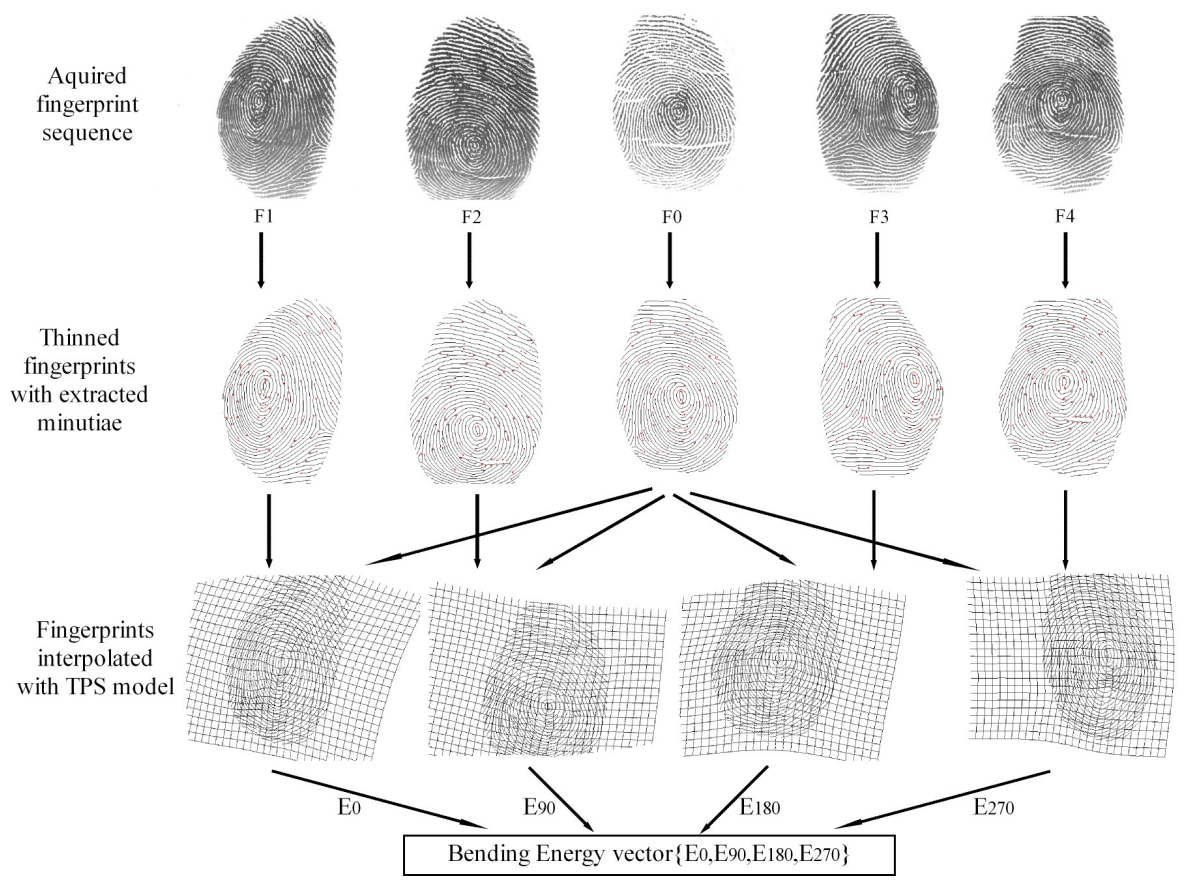

Fig. 2. The main steps of the bending energy vector computation

is captured and their minutiae are extracted from the thinned images. We respectively calculate the TPS models based on the paired landmarks between the natural fingerprint and the other four distorted fingerprints. The natural fingerprint F0 is distorted into the fingerprint skeletons in the third row by the corresponding TPS models. F0 is covered with griding before interpolating to represent the distortion of the TPS models clearly.

\subsection{Fuzzy Similarity Measure}

The fuzzy similarity based on bending energy vector method is applied to discriminate a real one from a fake finger. For an input finger, we compute the similarity between the current bending energy vector and the trained fuzzy feature set during the enrollment. The similarity score is in the range $[0,1]$, indicating how much the current finger is similar to the real one( 1 means real finger).

All elements in bending energy vector space of real finger construct real finger's fuzzy feature set $\tilde{V}$. We define $z_{r}$ as he center of fuzzy feature set:

$$
z_{r}=\frac{\Sigma_{v \in \tilde{V}} v}{\operatorname{Vol}(\tilde{V})}
$$

where $\operatorname{Vol}(\tilde{V})$ denotes the size of $\tilde{V}, z_{r}$ is essentially the mean of all elements of feature set. While averaging over all features in a feature set increases the robustness of fuzzy 
feature, at the same time, lots of useful information is submerged into the smoothing process because a set of feature vectors are mapped to a single feature vector.

Building or choosing a proper membership function is application dependent. The most commonly used prototype membership functions are cone, exponential, and Cauchy functions [7]. In the proposed algorithm, the modified Cauchy function is chosen due to its good expression and high-computational efficiency.

The membership function of bending energy vector $e$ to real finger's fuzzy feature set $\tilde{V}: C_{r}(e) \rightarrow[0,1]$, is defined as:

$$
C_{r}(e)= \begin{cases}1 & \text { if } h\left(e-z_{r}\right)=\text { True } \\ \frac{1}{1+\left(\frac{\left\|e-z_{r}\right\|}{m}\right)^{a}} & \text { otherwise }\end{cases}
$$

where $m$ and $a, m>0, a \geq 0, \boldsymbol{z}_{r}$ is the center location of the fuzzy set. $h\left(e-z_{r}\right)=$ True, if and only if the value of each entry in feature vector is less than the valve of corresponding entry in feature vector $z_{r} . m$ represents the width $\left\|e-z_{r}\right\|$ of the function, and $a$ determines the smoothness of the function. Generally, $m$ and $a$ describe the grade of fuzziness of the corresponding feature. It is obvious that the farther a feature vector moves away from the cluster center, the lower the degree of membership to the fuzzy feature.

Likewise, all elements in bending energy vector space of fake finger construct the fuzzy feature set $\tilde{U}$. We define the membership function of bending energy vector $e$ to fake finger's fuzzy feature set $\tilde{U}: C_{f}(e) \rightarrow[0,1]$.

Consequently, the similarity of the bending energy vector to the fuzzy feature sets is defined as $\operatorname{Sim}(e)=(1-p) * C_{r}(e)+p *\left(1-C_{f}(e)\right)$, where $p \in[0,1]$ adjusts the significance of $C_{r}(e)$ and $C_{f}(e)$.

\section{Experimental Results}

A database of fingerprint sequences is collected to evaluate the fake fingerprint detection approach. 120 fake fingers are manufactured (made of silicone AL20) starting from fingers of the 20 cooperating volunteers and ten sequences are recorded for each finger. We also capture the same-scaled fingerprint sequences from the corresponding real fingers. All the fingerprints are acquired through CrossMatch V300 (Optical sensor). The database is divided into two disjoint sets: the training set (20 real/fake fingers) used to select the parameters of the approach and the test set (100 real/fake fingers) used to measure the performance. The matching where the number of landmarks exceeds a certain threshold $n T h r$ (In our experiments $n T h r=15$ ) is taken into account so that the fake-detection performance is evaluated more accurately. Fig. 3 shows the distribution of the number of landmarks $N$ and the bending energy $E$ in fake and real fingerprint matching.

Let FAR be the proportion of transactions with a fake finger that are incorrectly accepted and FRR be the proportion of transactions with a real finger that are incorrectly rejected. The Equal Error Rate (EER) of the proposed approach measured in the above experiments is $4.5 \%$. Fig. 4 displays the performance diagram of our algorithm. 


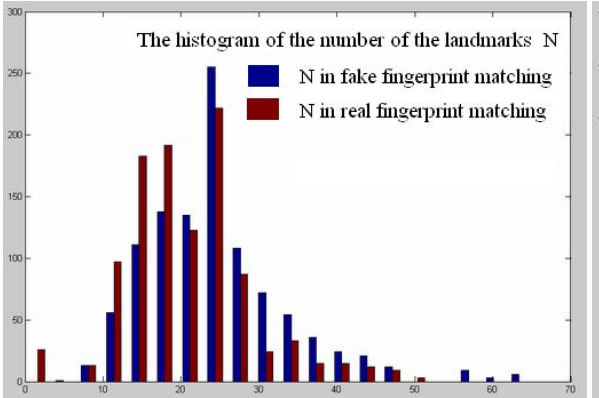

(a)

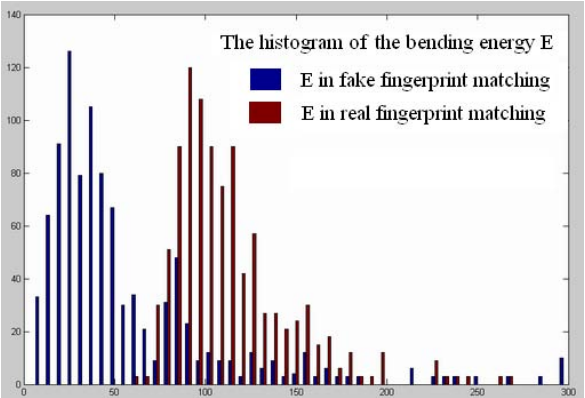

(b)

Fig. 3. The distribution of the number of landmarks and the bending energy

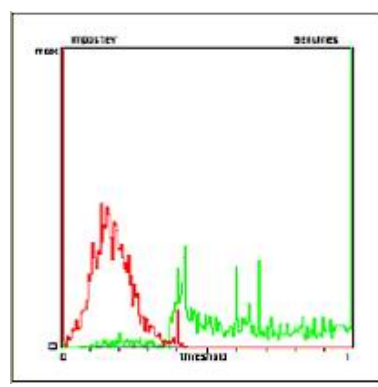

(a) Similarity distribution



(b) FNMR and FMR curve

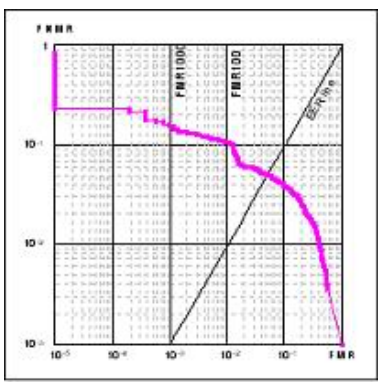

(c) ROC curve

Fig. 4. The performance of our algorithm (red curve is for fake fingers, green curve is for real ones)

The experimental results prove that when real fingers move on a scanner surface they mostly produce a larger distortion than the fake fingers. The proposed method is easily implemented without needing any additional hardware. It is based on normal steps of fingerprint identification, including matching the fingerprints and gaining their corresponding minutiae pairs. The decision is made based on the similarity of the input finger's bending energy vector to the bending energy fuzzy feature set so it is efficient. The experimental results on the database of real and fake fingers show that the performance of the method is available.

\section{Conclusion}

The distortion is one of the difficult problems in fingerprint recognition, but in this paper it is used to discriminate live finger from fake ones. The proposed method uses the thin-plate spline (TPS) model to realize fake fingers detection. The bending energy vector of the TPS model is used to determine the nature of the finger. For an input finger, we firstly compute the bending energy vector of the TPS model, then calculate 
the similarity of the bending energy vector to the trained bending energy fuzzy feature set. The experimental results on the database of real and fake fingers confirm that the performance of the method is very promising. Nevertheless, the performance of the method relies on the accuracy of minutiae extraction and pairing.

Acknowledgments. This paper is supported by the Project of National Science Fund for Distinguished Young Scholars of China (No. 60225008), the Key Project of National Natural Science Foundation of China (No. 60332010 and 60575007), the Project for Young Scientists' Fund of National Natural Science Foundation of China (No.60303022), and the Project of Natural Science Foundation of Beijing (No.4052026).

\section{References}

1. Derakhshani, R., Schuckers, S.A.C., Hornak, L.A., O'Gorman, L.: Determination of vitality from a non-invasive biomedical measurement for use in fingerprint scanners. Pattern Recognition 36(2), 383-396 (2003)

2. Antonelli, A., Cappelli, R., Maio, D., Maltoni, D.: A new approach to fake finger detection based on skin distortion. In: Zhang, D., Jain, A.K. (eds.) Advances in Biometrics. LNCS, vol. 3832, pp. 221-228. Springer, Heidelberg (2005)

3. Hong, L., Wan, Y., Jain, A.K.: Fingerprint image enhancement: algorithms and performance evaluation. IEEE Trans. Pattern Analysis Machine Intelligence 20(8), 777-789 (1998)

4. Chen, X.J., Tian, J., Yang, X.: An algorithm for distorted fingerprint matching based on local triangle features set. IEEE Trans. on Information, Forensics and Security 1(2) (2006)

5. Bazen, A.M., Gerez, S.H.: Fingerprint matching by thin-plate spline modelling of elastic deformations. Pattern Recognition 36(8), 1859-1867 (2003)

6. Ross, A., Dass, S., Jain, A.K.: A deformable model for fingerprint matching. Pattern Recognition 38(1), 95-103 (2005)

7. Hoppner, F., Klawonn, F., Kruse, R., Runkler, T.: Fuzzy cluster analysis: methods for classification, Data Analysis and Image Recognition. John Wiley \& Sons, Chichester (1999)

8. Bookstein, F.L.: Principal warps: Thin-plate splines and the decomposition of deformations. IEEE Transactions on Pattern Analysis and Machine Intelligence 2(6), 567-585 (1989)

9. Zhu, E., Yin, J.P., Zhang, G.M.: Fingerprint matching based on global alignment of multiple reference minutiae. Pattern Recognition 38(10), 1685-1694 (2005)

10. Matsumoto, T.H., Yamada, K., Hoshino, S.: Impact of Artificial 'Gummy' Fingers on. Fingerprint Systems. In: Proceedings of SPIE, vol. 4677 (2002)

11. Rohr, K., Fornefett, M., Stiehl, H.S.: Approximating Thin-Plate Splines for Elastic Registration: Integration of Landmark Errors and Orientation Attributes. In: Proceedings of the 16th International Conference on Information Processing in Medical Imaging, pp. 252-265 (1999) 